\title{
Impact of Medical Scribes in Primary Care on Productivity, Face-to-Face Time, and Patient Comfort
}

\author{
Leah Zallman, MD, MPH, Karen Finnegan, MPH, David Roll, MD, \\ Martina Todaro, MA, Rawan Oneiz, MD, and Assaad Sayah, MD
}

Background: Medical scribes are a clinical innovation increasingly being used in primary care. The impact of scribes in primary care remain unclear. We aimed to examine the impact of medical scribes on productivity, time spent facing the patient during the visit, and patient comfort with scribes in primary care.

Methods: We conducted a prospective observational pre-post study of 5 family and internal medicine-pediatrics physicians and their patients at an urban safety net health clinic. Medical scribes accompanied providers in the examination room and documented the clinical encounter. After an initial phase-in period, we added an additional 20 -minute patient slot per 200-minute session. We examined productivity by using electronic medical record data on the number of patients seen and work relative value units (work RVUs) per hour. We directly observed clinical encounters to measure the amount of time providers spent facing patients and other visit components. We queried patient comfort with scribes by using surveys administered after the visit.

Results: Work RVUs per hour increased by $10.5 \%$ from 2.59 prescribe to 2.86 post-scribe $(P<$ $.001)$. Patients seen per hour increased by $8.8 \%$ from 1.82 to $1.98(P<.001)$. Work RVUs per patient did not change. After scribe implementation, time spent facing the patient increased by $57 \%(P<.001)$ and time spent facing the computer decreased by $27 \%(P=.003)$. The proportion of the visit time that was spent face-to-face increased by $39 \%(P<.001)$. Most $(69 \%)$ patients reported feeling very comfortable with the scribe in the room, while the proportion feeling very comfortable with the number of people in the room decreased from $93 \%$ to $66 \%(P<.001)$.

Conclusions: Although the full implications of medical scribe implementation remain to be seen, this initial study highlights the promising opportunity of medical scribe implementation in primary care. (J Am Board Fam Med 2018;31:612-619.)

Keywords: Electronic Health Records, Physician-Patient Relations, Primary Health Care, Prospective Studies

Implementation of electronic medical records (EMRs) is associated with reductions in cost of care and improved quality ${ }^{1,2}$, leading to a 2014 federal government mandate on their widespread adop-

This article was externally peer reviewed.

Submitted 30 July 2017; revised 12 January 2018; accepted 23 January 2018.

From Institute for Community Health, Malden, MA (LZ, KF, MT); Cambridge Health Alliance, Cambridge (LZ, DR, AS); Harvard Medical School, Boston (LZ, DR, AS); Alpert Medical School Of Brown University, Providence, RI (RO)

Funding: none.

Conflict of interest: none declared.

Corresponding author: Leah Zallman, MD, MPH, 350

Main Street 4th floor, Malden MA 02148 (E-mail: lzallman@challiance.org). tion. However, concerns have arisen about the impact of EMRs on patient-physician relationships ${ }^{3-5}$ and on the clerical burden for providers. ${ }^{6-8}$ Concerns regarding the impact of EMR use on patientphysician relationships have centered on the computer's negative influence on patient centeredness during the clinical encounter. ${ }^{3-5}$ In addition, an unintended consequence of EMRs has been an increase in the clerical burden for practitioners, particularly for primary care providers (PCPs). ${ }^{6,8}$ EMRs allow providers to document encounters, review testing results, consult with team members and each other, and communicate directly with patients via patient portals. PCPs have become re- 
sponsible for a significant amount of data entry, leading to a large amount of time spent in documenting and navigating these records. Combined with a national shortage of primary care physicians ${ }^{9}$ and increasing physician burnout ${ }^{10}$, these concerns have catalyzed efforts to redistribute responsibilities in an expanded primary care team. ${ }^{11}$

Medical scribes are an innovation that is increasingly used to address these challenges in primary care. Medical scribes are unlicensed individuals who are present with the provider in a patient's room and record key elements of the encounter. Some studies have described the impact of team members who assist with documentation in addition to other patient care responsibilities. ${ }^{12-15} \mathrm{We}$ focused on medical scribes, whose principal role is to improve the efficiency of provider documentation in the EMR, thus facilitating higher productivity for providers (and whom do not participate in other aspects of patient care). Indeed, implementation of medical scribes in emergency departments and outpatient specialty practices (ie, cardiology and urology) has been associated with increased productivity. ${ }^{16,17}$ More recent data from primary care practices have demonstrated improved productivity ${ }^{18}$ and decreased documentation time. ${ }^{18-20}$ However, one of these studies featured implementation of a note template at the same time as scribe implementation, raising the possibility that improvements were due to note templates. ${ }^{20}$ The others were a quality improvement study not designed to create generalizable knowledge ${ }^{18}$ and a research study that measured satisfaction with documentation time as a proxy for documentation time. ${ }^{19}$ Thus, the impact of medical scribes on PCP productivity remains unclear.

In addition to improving productivity, medical scribes may also address another unintended consequences of the EMR-reductions in patient centeredness. That is, scribes may allow providers to face their patients, rather than the computer, thus restoring some aspects of the patient-provider relationship. At the same time, discomfort with an additional person in the examination room could detract from the patient-provider relationship for some patients. ${ }^{21}$ Indeed, the only study examining time spent facing the patient, conducted in a cardiology clinic, found that providers spent more time facing the patient. ${ }^{16}$ Data regarding the impact on patient satisfaction are more mixed, with some studies reporting improvement in patient sat- isfaction $^{22}$, some reporting no change on all or most measures ${ }^{17,19,23}$, and some reporting reductions. ${ }^{20}$ Only 2 of these studies were conducted in primary care ${ }^{19,20}$, of which 1 reported a high degree of comfort with scribes. ${ }^{20}$

Given the recent increase in medical scribe use in primary care, we aimed to add to the literature by providing data that help elucidate the impact of medical scribes on productivity, time spent facing the patient during the visit, and patient comfort with scribes.

\section{Methods}

\section{Setting}

We conducted this study at a safety net urban primary care clinic between March 2016 and April 2017. We included 5 family and internal medicine physicians and their patients. Physicians use an integrated EMR software to capture patient visit data. This study was approved by the Cambridge Health Alliance Institutional Review Board.

\section{Description of Intervention}

A professional scribe company provided medical scribe services starting in October 2016. A total of 7 scribes supported the 5 physicians. Most scribes in our practice were upper-level undergraduates or recent college graduates in a "gap year," with a goal of entering medical school or other health professions (such as physician assistant or physical therapy). Scribes received 60 hours of training by the scribe company and 40 hours of training in the clinic, learning about the specific EMR and clinic flow. All physicians in the practice were invited to use a scribe, and all agreed to participate. The physicians had a range of 1 year to 10 years in clinical practice. Two physicians practiced halftime and 3 practiced full-time or nearly full-time. All scribes and physicians were proficient in English only.

Our scribes primarily helped the physicians to document the clinical encounter, which the physicians reviewed and edited. Scribes typically participated in the pre-session huddle with the physician and medical assistant in which the objectives for the session and health maintenance items are reviewed. The EMR access for scribes was built in such a way so that they could pend orders for providers to sign, but the physicians rarely took advantage of this ability. 
The physicians were assigned scribes for all sessions they were in the clinic; due to absences and vacations, scribes were present for approximately $90 \%$ of sessions. To the extent possible, physicians were paired with a primary scribe; when the primary scribe was not available, physicians occasionally worked with alternate scribes. After an initial phase-in period, we added an additional 20-minute patient slot per 200-minute session to each provider's schedule in March 2017. At the time of follow-up data collection, providers had worked with scribes for 1 month to 6 months.

\section{Data Collection}

\section{Productivity}

To determine the impact of the scribe intervention on provider productivity, we extracted the number of patients seen and work relative value units (work RVUs) per hour from the EMR. RVUs provide an estimate of provider productivity during clinical sessions. We collected these data for each hour of scheduled patient visits during a preintervention period (August-September 2016) and postintervention period (March-April 2017). We were unable to remove the estimated $10 \%$ of postscribe visits that did not have a scribe. In addition, we extracted the proportion of charts closed by the end of the day (11:59 PM) from the EMR. Because productivity can vary by season, we wanted to confirm that changes persisted in RVUs and patients per hour even when comparing the same season in the year before. In this analysis, we compared March-April 2017 to March-April 2016. If changes did not persist in this analysis, we would be concerned that seasonal variation in productivity between AugustSeptember and March-April accounted for changes in productivity.

\section{Face-to-Face Time and Other Cycle Components}

For face-to-face and other cycle components, we collected data primarily through direct observation of providers. We collected all post-scribe observation data for visits in which a scribe was present. We defined cycle time as the time it took for the provider to prepare for the visit, complete the visit, and finish the chart for each patient. We defined pre-visit time as any time spent reviewing the chart before entering the examination room. We defined visit time as the time that the provider spent in the clinical examination room. During the visit, we tracked our primary outcome, face-to-face time, as the time the physician spent interacting with the patient or family member, without using or looking at the computer. We considered the difference between face-to-face time and the visit time to be time spent facing the computer. We defined postvisit time as any time spent in the chart after leaving the examination room until the chart was closed. To track post-visit time, we employed 2 methodologies: direct observation for charting during business hours and physician self-timing using phonebased or handheld timers for charting after the business day. We summed pre-visit, visit, and postvisit time to estimate the total provider cycle time.

\section{Patient Comfort}

We administered surveys to patients or family members aged 18 years and older in 5 languages (English, Arabic, Haitian Creole, Portuguese, and Spanish) after the visit. We collected all post-scribe surveys for visits in which a scribe was present. Because there were no standardized instruments to assess level of comfort with scribes, we created questions based on our experience. The survey asked patients about comfort with the scribe and the number of people in the room by using a 4-point Likert scale (not at all comfortable, somewhat uncomfortable, somewhat comfortable, very comfortable). Surveys were translated by certified medical interpreters.

\section{Statistical Analyses}

\section{Productivity}

We extracted productivity data from the EMR. We calculated productivity as mean RVUs and mean number of patients seen per scheduled hour. As others have done ${ }^{18}$, our institution considered an additional 20 -minute $(10 \%)$ patient slot per 200minute session as a significant change. Thus, we considered a $10 \%$ change in productivity as clinically significant. Our study was powered to detect a $10 \%$ difference in visits per hour (or 0.18 visits per hour) and a $6 \%$ difference (or 0.16 RVUs per hour) in RVUs per hour, with an $\alpha$ of 0.05 . It therefore met or exceeded the power level necessary to detect a clinically significant change. We compared mean productivity for 980 hours in the pre-scribe period and 718 hours in the post-scribe period by using a $t$ test. We compared proportion of visits closed by the end of the day (11:59 PM) using a $\chi^{2}$ test of association. 
Face-to-Face Time and Other Cycle Components

We collected face-to-face time and other cycle components data through observation. We examined 92 pre-scribe visits and 29 post-scribe visits. We are not aware of published studies articulating a rationale for a clinically significant change in face-to-face time for PCPs. In the absence of this rationale, we relied on clinical experience. Based on the clinical experience of 3 physicians on the study team (DR, AS, LZ), we considered an increase of face-to-face time of $50 \%$ to be clinically significant. Our study was powered to detect a difference of 2:49 minutes ( $46 \%$ increase) of face-to-face time with an $\alpha$ of 0.05 . It therefore met the power level necessary to detect a clinically significant change. We calculated mean face-to-face time and other cycle components for each patient visit for 4 providers. We excluded 1 provider from analysis due to outlying observations based on interquartile range. We compared pre-values to post-values by using a $t$ test with a Satterthwaite approximation to account for unequal variance when necessary.

\section{Patient Comfort with Scribes}

We collected data on patient comfort by using selfadministered surveys (100 pre-scribe and 81 postscribe). We collected all post-scribe patient surveys for visits in which scribes were used. We are not aware of published studies articulating a rationale for a clinically significant change in patient comfort level with the number of people in the room. In the absence of this rationale, we relied on clinical experience. Based on the clinical experience of 3 physicians on the study team (DR, AS, LZ), we considered a $20 \%$ decrease in comfort level with the number of people in the room to be clinically significant. Our study was powered to detect an $83 \%$ difference with an $\alpha$ of 0.05 , thus not meeting the power level nec- essary to detect a clinically significant change. We dichotomized comfort with number of people in the room and comfort with the scribe into 2 categories (very comfortable versus all others) to capture a meaningful target of high levels of comfort. We compared comfort with the number of people in the room before and after scribes with a $\chi^{2}$ test of association.

\section{Results}

\section{Productivity}

Both RVUs per hour and patients per hour increased from pre-scribe to post-scribe (Table 1). RVUs per hour increased by $10.5 \%$ from 2.59 prescribe to 2.86 postscribe $(P<.001)$. Patients seen per hour increased by $8.8 \%$ from 1.82 to 1.98 $(P<.001)$. RVUs per patient did not change. The proportion of charts closed by the end of the day (by 11:59 PM) did not change significantly.

\section{Seasonal Analysis}

We found no significant differences in productivity comparing March-April 2017 to March-April 2016, suggesting seasonal variation does not account for these findings.

\section{Face-to-Face Time and Other Cycle Components}

After scribe implementation, face-to-face time increased by $57 \%(P<.001)$ and computer time decreased by $27 \%(P<.001)$ (Table 2$)$. The proportion of the visit time that was spent face-to-face increased by $39 \%(P<.001)$. There were no significant changes in visit time, previsit, postvisit, or total provider cycle time.

\section{Patient Comfort with Scribes}

Patients who completed the survey before scribes were $37 \%$ male, $31 \%$ Hispanic, and 50.6 years old. Patients who completed the surveys postscribe

Table 1. Productivity and Charts Closed by End of Day Before (August-September 2016) and After (March-April 2017) Implementing Scribes in Primary Care

\begin{tabular}{lccc}
\hline & $\begin{array}{c}\text { Pre-scribe } \\
\mathrm{n}=980 \text { hours } \\
\text { Mean (SD) }\end{array}$ & $\begin{array}{c}\text { Post-scribe } \\
\mathrm{n}=718 \text { hours } \\
\text { Mean (SD) }\end{array}$ & $P$ value, $t$ test \\
\hline RVUs/h & $2.59(1.17)$ & $2.86(1.22)$ & $<.001$ \\
Patients/h & $1.82(0.76)$ & $1.98(0.78)$ & $<.001$ \\
RVUs/patient & $1.42(0.29)$ & $1.44(0.29)$ & .37 \\
Proportion of charts closed by end of day (11:59 PM) & $0.67(0.42)$ & $0.71(0.40)$ & .37 \\
\hline
\end{tabular}

RVU, relative value unit; SD, standard deviation. 
Table 2. Face-to-Face Time and Other Cycle Components Before (August-September 2016) and After (March-April 2017) Implementing Scribes in Primary Care*

\begin{tabular}{lcrc}
\hline & $\begin{array}{c}\text { Pre-scribe } \\
\mathrm{n}=92\end{array}$ & $\begin{array}{c}\text { Post-scribe } \\
\mathrm{n}=29\end{array}$ & $P$ value $t$ test \\
& Minutes:second (SD) & Minutes:second (SD) & .44 \\
\hline Pre-visit & $2: 09(1: 55)$ & $2: 33(2: 36)$ & .15 \\
Visit & $17: 40(6: 44)$ & $19: 46(6: 39)$ & $<.001$ \\
$\quad$ Computer & $9: 27(4: 16)$ & $6: 53(2: 51)$ & $<.001$ \\
$\quad$ Face-to-face & $8: 13(4: 41)$ & $12: 53(5: 28)$ & $<.001$ \\
Proportion of visit time spent face to face & $0.46(0.16)$ & $0.64(0.12)$ & .47 \\
Post-visit & $5: 27(3: 54)$ & $4: 53(2: 49)$ & .31 \\
Total provider cycle time & $25: 16(8: 42)$ & $27: 12(9: 20)$ & \\
\hline
\end{tabular}

*Other cycle complements include pre-visit, visit, computer, and post-visit time.

Pre-visit time $=$ time spent reviewing the chart prior to entering the exam room; visit time $=$ time the provider spent in the clinical exam room; face-to-face time = time the physician spent interacting with the patient or family member, without using or looking at the computer; computer time $=$ difference between face-to-face time and visit time; post-visit time $=$ time spent in the chart after leaving the exam room until the chart was closed; total provider cycle $=$ sum of pre-visit, visit and post-visit time.

$\mathrm{SD}$, standard deviation.

were $25 \%$ male, $37 \%$ Hispanic, and 45.6 years old. (Appendix B).

Although two-thirds of patients reported being very comfortable with having the scribe in the room, the proportion of individuals who felt very comfortable with the number of people in the room decreased significantly from $93 \%$ to $66 \%(P<$ .0001) (Table 3). There was no significant difference in being very comfortable with scribes among visits that used interpreters (12\% of visits) compared with those without $(P=.27$, data not shown). However, all the interpretation in this context was performed via telephone or video modalities.

\section{Discussion}

Medical scribes were associated with increased productivity and increased time and proportion of visit spent facing patients during the clinical encounter in a safety net primary care clinic. Although the comfort level with having the scribe in the room was very high, the level of comfort with the number of people in the room decreased.
This is the first research study examining the impact of scribes on productivity in primary care. We found no prior examinations of the impact on work RVUs per hour and the only prior data on patients per hour is from a feasibility study that was not designed to provide generalizable knowledge (and had no statistical analyses). ${ }^{18}$ Our finding is consistent with data from other outpatient settings such as cardiology and urology practices that demonstrate increased productivity. ${ }^{17,24} \mathrm{In}$ addition to providing evidence that medical scribe implementation improves productivity, our study provides insight on the mechanism for this increase. Medical scribes could increase productivity through 2 mechanisms: by allowing more patients to be seen in the same amount of time and by supporting documentation that allows the provider to bill at higher rates per patient. We found that RVUs per patient did not increase, suggesting that the mechanism for increased productivity was the availability of an additional patient slot per session.

Table 3. Patient Comfort with Scribes and the Number of People in the Room Before (August-September 2016) and After (March-April 2017) Implementing Scribes in Primary Care

\begin{tabular}{|c|c|c|c|}
\hline Comfort of patients & $\begin{array}{c}\text { Pre-scribe } \\
\mathrm{n}=100 \\
\text { Very comfortable, \% }\end{array}$ & $\begin{array}{c}\text { Post-scribe } \\
\mathrm{n}=81 \\
\text { Very comfortable, \% }\end{array}$ & $P$ value, $\chi^{2}$ \\
\hline Comfort with number of people in room & 93 & 66 & $<.0001$ \\
\hline Comfort with scribe & & 69 & \\
\hline
\end{tabular}


The growth of population health, meaningful use, and global payment initiatives will likely exacerbate the PCP workload and associated clerical burden. Thus, examination of metrics relevant to PCPs is critical. Indeed, examination of medical scribes as a mechanism for relieving provider burnout has been suggested by leading burnout experts. ${ }^{6}$ Although increased productivity is one of the most compelling drivers for this model, an important balancing measure is the amount of time providers spend per patient. In our study, productivity increased, while the proportion of charts closed by the end of the day did not change significantly. Prior studies report improvement in provider perceptions of their charting time, ${ }^{19}$ improved self-perceived ability to stay on schedule, ${ }^{20}$ and improved satisfaction with the amount of time documenting. ${ }^{19}$ Thus, further exploration of the impact of scribes on cycle time in primary care is warranted.

In addition to charting time and cycle time, other provider metrics such as provider satisfaction and burnout are important metrics. Indeed, PCPs report high levels of comfort with scribes, ${ }^{18}$ that scribes are helpful, ${ }^{18}$ and that more time facing the patient is a positive aspect of working with scribes. ${ }^{18,20,25}$ In light of these data, our finding that providers spend more time facing patients suggests that scribes have the potential to improve the provider experience. However, the mechanism for the impact of scribes on PCP experience is likely to be multifactorial. Thus, more exploration on the impact of medical scribes on PCP satisfaction is warranted. In addition, as reimbursement shifts from fee-for-service to global-payment models, a full assessment of scribes' financial impact should assess their ability to help providers meet contract performance metrics, code for high-risk conditions, and increase panel size.

Patient centeredness is important to patients, health systems, and providers alike. Studies examining patient satisfaction in primary care have demonstrated no change in patient satisfaction, ${ }^{19}$ or small but insignificant decreases in satisfaction levels in primary care. ${ }^{20}$ These studies are inconsistent with a prior qualitative study reporting perceived increased attention from PCPs. ${ }^{14}$ This equipoise may in fact reflect that patient perceptions of attentiveness and satisfaction with care may be driven by multiple factors. Our study may add a new perspective. Consistent with other literature, we found that comfort level with scribes was high. ${ }^{20}$ However, we found that the level of comfort with the number of people in the room decreased after scribe implementation, shedding light on a possible mechanism for why patient satisfaction may not improve in primary care. This decrease in comfort with the number of people in the room may reflect the context of our study. Although medical interpretation is available, patients with limited English proficiency often bring family members to assist in navigating the system. Indeed in our safety net setting, additional family members or friends were common; before scribe implementation, $47 \%$ of patients reported at least 1 person besides themselves and the doctor were in the room and 19\% reported at least 2 . The degree to which changes in comfort with the number of people in the room after scribe implementation is affected by the number of people already present in visits remains an interesting area for further inquiry.

These data must be interpreted in the context of several limitations. Our study represents the experience of a limited number of providers at a single institution. Our method of using scribes may differ from methods in other settings, and this may alter the impact of scribes at different sites. Although the prospective design limits bias, the study is observational and therefore susceptible to influence from various unobserved factors. Physicians may not have been observed on a day when they were working with their primary scribe. This may have led to an underrepresentation of the full effect of scribe implementation. Our postscribe productivity data include the estimated $10 \%$ of visits that did not have a scribe. This too may have led to an underrepresentation of the full effect of scribe implementation. Small sample sizes may have limited our ability to detect changes for some outcomes. Further larger studies will help confirm our findings. Though not a primary outcome, we tracked time spent charting after the visit, with a combination of observations and self-reported data, which may account for the relatively low postvisit charting times we recorded. We used this method both pre-scribe and post-scribe, thus limiting the likelihood that inaccuracies accounted for any changes in outcomes. Nonetheless, the reliability of these time estimates was not verified and they may be subject to bias.

Nonetheless, our study is the first to demonstrate increased productivity and time spent facing patients after medical scribe implementation in primary care. 
These increases in productivity were accompanied by a high level of comfort with having the scribe in the room and decreased level of comfort with the number of people in the room after scribe implementation. This study was limited in size and scope, and the effects of this innovation on physicians, patients, and health care systems require further investigation. Although the full implications of medical scribe implementation remain to be seen, this initial study highlights the promising opportunity of medical scribe implementation in primary care.

The authors thank Loubaba Riahy and Stephen Dolat for their help in coordinating data collection and in helping us interpret this data. We would also like to thank the patients and physicians who participated in this study.

To see this article online, please go to: http://jabfm.org/content/ 31/4/612.full.

\section{References}

1. Buntin MB, Burke MF, Hoaglin MC, Blumenthal D. The benefits of health information technology: a review of the recent literature shows predominantly positive results. Health Aff (Millwood) 2011;30:464-71.

2. Kern LM, Barron Y, Dhopeshwarkar RV, Edwards A, Kaushal R. Electronic health records and ambulatory quality of care. J Gen Intern Med 2013;28:496-503.

3. Crampton NH, Reis S, Shachak A. Computers in the clinical encounter: a scoping review and thematic analysis. J Am Med Inform Assoc 2016;23:654-65.

4. Makoul G, Curry RH, Tang PC. The use of electronic medical records: communication patterns in outpatient encounters. J Am Med Inform Assoc 2001;8:610-5.

5. Shachak A, Reis S. The impact of electronic medical records on patient-doctor communication during consultation: a narrative literature review. J Eval Clin Pract 2009;15:641-9.

6. Shanafelt TD, Dyrbye LN, West CP. Addressing physician burnout: the way forward. JAMA 2017; 317:901-2.

7. Sinsky C, Colligan L, Li L, et al. Allocation of physician time in ambulatory practice: a time and motion study in 4 specialties. Ann Intern Med 2016; 165:753-60.

8. Sinsky CA. Designing and regulating wisely: removing barriers to joy in practice. Ann Intern Med 2017; 166:677-8.

9. Petterson SM, Liaw WR, Phillips RL, Jr., Rabin DL, Meyers DS, Bazemore AW. Projecting US primary care physician workforce needs: 2010-25. Ann Fam Med 2012;10:503-9.

10. Shanafelt TD, Boone S, Tan L, et al. Burnout and satisfaction with work-life balance among US physi- cians relative to the general US population. Arch Intern Med 2012;172:1377-85.

11. Bodenheimer T, Pham HH. Primary care: current problems and proposed solutions. Health Aff (Millwood) 2010;29:799-805.

12. Ammann H, Hele' K, Salibi N, Wilcox S, Cohen M. Adapting EHR scribe model to community health centers: the experience of Shasta Community Health Center's pilot. San Francisco (CA): Blue Shield of California Foundation; 2012.

13. Anderson P, Halley MD. A new approach to making your doctor-nurse team more productive. Fam Pract Manag 2008;15:35-40.

14. Blash L, Dower C, S C. University of Utah community clinics-medical assistant teams enhance patientcentered, physician-efficient care. San Francisco, CA: Center for the Health Professions, University of California; 2011.

15. Reuben DB, Knudsen J, Senelick W, Glazier E, Koretz BK. The effect of a physician partner program on physician efficiency and patient satisfaction. JAMA Intern Med 2014;174:1190-3.

16. Bank A, Obetz C, Konrardy A, et al. Impact of scribes on patient interaction, productivity, and revenue in a cardiology clinic: a prospective study. Clinicoecon Outcomes Res 2013;5:399-406.

17. Koshy S, Feustel PJ, Hong M, Kogan BA. Scribes in an ambulatory urology practice: patient and physician satisfaction. J Urol 2010;184:258-62.

18. Earls S, Savageau J, Begley S, Saver B, Sullivan K, Chuman A. Can scribes boost FPs' efficiency and job satisfaction? J Fam Pract 2017;66:206-14.

19. Gidwani R, Nguyen C, Kofoed A, et al. Impact of scribes on physician satisfaction. Patient satisfaction, and charting efficiency: a randomized controlled trial. Ann Fam Med 2017;15:427-33.

20. Imdieke BH, Martel ML. Integration of medical scribes in the primary care setting: improving satisfaction. J Ambul Care Manage 2017;40:17-25.

21. Schiff GD, Zucker L. Medical scribes: salvation for primary care or workaround for poor EMR usability? J Gen Intern Med 2016;31:979-81.

22. Bastani A, Shaqiri B, Palomba K, Bananno D, Anderson $W$. An ED scribe program is able to improve throughput time and patient satisfaction. Am J Emerg Med 2014;32:399-402.

23. Shuaib W, Hilmi J, Caballero J, et al. Impact of a scribe program on patient throughput, physician productivity, and patient satisfaction in a community-based emergency department. Health Informatics J 2017;1460458217704255.

24. Bank AJ, Gage RM. Annual impact of scribes on physician productivity and revenue in a cardiology clinic. Clinicoecon Outcomes Res 2015;7:489-95.

25. Yan C, Rose S, Rothberg MB, Mercer MB, Goodman K, Misra-Hebert AD. Physician, scribe, and patient perspectives on clinical scribes in primary care. J Gen Intern Med 2016;31:990-5. 
Appendix A. Seasonal Analysis for Productivity and Charts Closed by End of Day Before (March-April 2016) and After (March-April 2017) Implementing Scribes in Primary Care*

\begin{tabular}{lccc}
\hline & $\begin{array}{c}\text { Pre-scribe } \\
\mathrm{n}=980 \text { hours } \\
\text { Mean (SD) }\end{array}$ & $\begin{array}{c}\text { Post-scribe } \\
\mathrm{n}=718 \text { hours } \\
\text { Mean (SD) }\end{array}$ & $P$ value, $t$ test \\
\hline Relative value units/h & $2.71(1.21)$ & $2.86(1.22)$ & .02 \\
Patients/h & $1.86(0.50)$ & $1.98(0.78)$ & $<.001$ \\
Proportion of charts closed by end of day (11:59 PM) & $0.67(0.42)$ & $0.71(0.40)$ & .35 \\
\hline
\end{tabular}

*To examine the possibility that seasonal variation contributed to our findings, we compared March-April 2016 with March-April 2017. Because productivity can vary by season, we wanted to confirm that changes persisted even when comparing the same season in the year before. If changes did not persist in this analysis, we would be concerned that seasonal variation in productivity between August-September and March-April accounted for changes in productivity.

Appendix B. Characteristics of Patients Who

Completed Satisfaction Surveys Before (August-

September 2016) and After (March-April 2017)

Implementing Scribes in Primary Care

\begin{tabular}{lccc}
\hline & $\begin{array}{c}\text { Pre-scribe, } \\
\mathrm{n}=100\end{array}$ & $\begin{array}{c}\text { Post-scribe, } \\
\mathrm{n}=81\end{array}$ & $P$ value \\
\hline $\begin{array}{l}\text { Age, mean (SD) } \\
\text { Sex }\end{array}$ & $50.63(18.25)$ & $45.61(16.37)$ & .05 \\
$\quad$ Male & $37 \%$ & $25 \%$ & .09 \\
$\quad$ Female & $63 \%$ & $75 \%$ & \\
Hispanic & & & \\
Yes & $31 \%$ & $37 \%$ & .39 \\
No & $69 \%$ & $63 \%$ & \\
\hline
\end{tabular}

SD, standard deviation. 